\title{
Local- and regional-scale racial and ethnic disparities in air pollution determined by long-term mobile monitoring
}

\author{
Sarah E. Chambliss ${ }^{a}{ }^{\circ}$, Carlos P.R. Pinon $^{a}$, Kyle P. Messier ${ }^{b}{ }^{\circ}$, Brian LaFranchi ${ }^{c}$, Crystal Romeo Upperman ${ }^{(}{ }^{\circ}$,

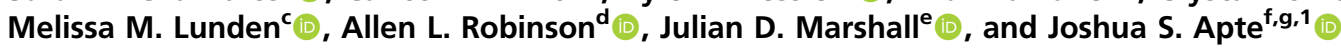 \\ ${ }^{a}$ Department of Civil, Architectural and Environmental Engineering, University of Texas at Austin, Austin, TX 78712; ${ }^{b}$ National Toxicology Program, \\ National Institute of Environmental Health Sciences, Durham, NC 27713; 'Aclima, Inc., San Francisco, CA 94111; ${ }^{\mathrm{d} D e p a r t m e n t}$ of Mechanical Engineering, \\ Carnegie Mellon University, Pittsburgh, PA 15213; 'Department of Civil and Environmental Engineering, University of Washington, Seattle, WA 98195; \\ fDepartment of Civil and Environmental Engineering, University of California, Berkeley, CA 94720; and ${ }^{9}$ School of Public Health, University of California, \\ Berkeley, CA 94720
}

Edited by Susan Hanson, Clark University, Worcester, MA, and approved July 26, 2021 (received for review May 18, 2021)

Disparity in air pollution exposure arises from variation at multiple spatial scales: along urban-to-rural gradients, between individual cities within a metropolitan region, within individual neighborhoods, and between city blocks. Here, we improve on existing capabilities to systematically compare urban variation at several scales, from hyperlocal $(<100 \mathrm{~m})$ to regional $(>10 \mathrm{~km})$, and to assess consequences for outdoor air pollution experienced by residents of different races and ethnicities, by creating a set of uniquely extensive and high-resolution observations of spatially variable pollutants: $\mathrm{NO}, \mathrm{NO}_{2}$, black carbon (BC), and ultrafine particles (UFP). We conducted full-coverage monitoring of a wide sample of urban and suburban neighborhoods (93 $\mathrm{km}^{2}$ and 450,000 residents) in four counties of the San Francisco Bay Area using Google Street View cars equipped with the Aclima mobile platform. Comparing scales of variation across the sampled population, greater differences arise from localized pollution gradients for BC and NO (pollutants dominated by primary sources) and from regional gradients for UFP and $\mathrm{NO}_{2}$ (pollutants dominated by secondary contributions). Median concentrations of UFP, $\mathrm{NO}$, and $\mathrm{NO}_{2}$ are, for Hispanic and Black populations, 8 to $30 \%$ higher than the population average; for White populations, average exposures to these pollutants are 9 to $14 \%$ lower than the population average. Systematic racial/ethnic disparities are influenced by regional concentration gradients due to sharp contrasts in demographic composition among cities and urban districts, while within-group extremes arise from local peaks. Our results illustrate how detailed and extensive fine-scale pollution observations can add new insights about differences and disparities in air pollution exposures at the population scale.

air pollution | environmental justice | air quality

A ir pollution varies in complex patterns across the urban landscape, arising from the interplay of emissions source locations and atmospheric transport and transformation. Gradients exist at multiple spatial scales, reflecting regional, city-level, and neighborhood-level phenomena, including highly localized peaks near major sources (1-3). The uneven distribution of sources has been shown in the United States to cause systematically higher outdoor concentrations for people of color and communities facing disproportionate socioeconomic and environmental stressors (4-10). Increased air pollution exposure is associated with premature mortality and a multitude of chronic health problems, as well as increased vulnerability to extreme events such as wildfire pollution episodes and COVID-19 (11-13). Measurement and analysis of this disparity in outdoor concentrations are vital for understanding how the causes of air pollution (e.g., city zoning, infrastructure development, emissions sources) affect differential health outcomes. This understanding can aid in designing effective environmental justice measures and tracking the effects of the evolving urban landscape on population-wide and community exposure. Here, we use mobile monitoring (in-motion measurements by vehicle-mounted instruments) to observe highly localized air pollution patterns in a variety of urban settings and consider the implications for the measurement and mitigation of pollution exposure and environmental inequity.

The full complexity of multiscale patterns of air pollution is largely unknown in most urban areas despite great advances in measurement and modeling methods over the past few decades. Regulatory monitoring sites are sparsely distributed and generally do not measure unregulated pollutants of health concern, such as black carbon (BC) and ultrafine particles (UFP). While satellite remote sensing provides nearly global spatial coverage, most conventional products are limited in resolution to 1 to $5 \mathrm{~km}^{2}$ and do not include all pollutants of interest $(14,15)$. Mechanistic models predict concentrations over broad domains but are limited by computational constraints and data gaps (16). Recent statistical models provide both high spatial resolution and geographic coverage, but concentration predictions reflect generalized patterns, tend to predict central tendencies better than

\section{Significance}

It is known, to researchers and heavily impacted communities, that people of color face a higher average burden of air pollution. It was unknown whether racial/ethnic disparities were caused by spatial heterogeneities at the level of city blocks, neighborhoods, or urban regions. Our approach leverages a unique set of highly local observations, covering every city block of 13 cities and urban districts that are home to 450,000 people. We find that even for pollutants with steep localized gradients, differences in average outdoor concentrations among racial/ethnic groups are driven by regional variability. However, localized peaks indicate opportunities to reduce extremes within groups. The methods and findings of this study can inform strategies to reduce disparities in urban air pollution exposure.

Author contributions: S.E.C., A.L.R., J.D.M., and J.S.A designed research S.E.C, C.P.R.P. K.P.M., B.L., and M.M.L. performed research; S.E.C., K.P.M., B.L., M.M.L., and J.S.A. contributed new reagents/analytic tools; S.E.C., C.P.R.P., K.P.M., B.L., C.R.U., and M.M.L. analyzed data; and S.E.C., C.P.R.P., K.P.M., B.L., C.R.U., M.M.L., A.L.R., J.D.M., and J.S.A. wrote the paper.

Competing interest statement: B.L., C.R.U., and M.M.L. are employed by Aclima, Inc., which contributed to the collection of the measurements employed here.

This article is a PNAS Direct Submission.

This open access article is distributed under Creative Commons Attribution-NonCommercialNoDerivatives License 4.0 (CC BY-NC-ND).

${ }^{1}$ To whom correspondence may be addressed. Email: apte@berkeley.edu.

This article contains supporting information online at https://www.pnas.org/lookup/suppl/ doi:10.1073/pnas.2109249118/-/DCSupplemental.

Published September 7, 2021. 
extremes, and may miss local idiosyncrasies, especially for models with broad (e.g., national) domains (17). Despite the growing sophistication of these technologies, in many cases they are best suited to depict patterns of pollutants that are predominantly secondary, such as ozone and $\mathrm{PM}_{2.5}$, which tend to vary more over the regional than neighborhood scale (17). In contrast, fine-scale gradients dominate spatial variability for directly emitted (primary) pollutants like $\mathrm{BC}$ and for pollutants with highly localized transformation dynamics like UFP and NO $(1,18-22)$. Both BC and UFP are suspected to cause distinct health impacts (23-25), but these effects-and the potential racial/ethnic and socioeconomic health disparities-will remain poorly understood until higher-resolution measurements are more widespread $(25,26)$.

Mobile monitoring and low-cost sensors are increasingly used to detect fine-scale pollution gradients with applications ranging from new risk estimates for cardiovascular disease to the identification of unexpected sources of exposure disparity $(24,27)$. Low-cost sensors are used to supplement the density of existing regulatory networks, but appropriate sensors are not available for all pollutants and operation is limited by the capacity for consistent calibration and maintenance (28). Mobile monitoring has been used to measure multipollutant gradients in a wide range of urban contexts (29-31). Example applications of mobile monitoring include studies of short-range multipollutant variation attributable to highway traffic (31-35), infrastructure geometry, such as street canyons and near-road barriers $(22,36-38)$, and a variety of specific local sources $(37,39-41)$. While mobile monitoring is a flexible method, it is also labor intensive, requiring many repeated visits to collect enough localized measurements to capture the full temporal variation in conditions.

Because of the large resource requirement for long-term mobile monitoring, only a small number of campaigns have measured multiyear, high-resolution patterns over multiple neighborhoods $(19,42)$. Few have attempted comprehensive coverage of all major and residential streets of several contiguous neighborhoods $(21$, $41,43)$. This study presents the results of 32 mo of mobile monitoring along every street of 13 cities, towns, and urban districts $\left(93 \mathrm{~km}^{2}\right)$ distributed through four counties of the San Francisco Bay Area, providing over 2,100 h of sampling of four pollutants at $\sim 0.01 \mathrm{~km}^{2}$ (i.e., $\sim 100 \times 100 \mathrm{~m}^{2}$ ). We employ two custom-equipped Google Street View cars to repeatedly measure city block air quality, providing estimates of outdoor air pollution for a year2010 population of $\sim 450,000$ individuals and an opportunity to characterize, quantify, and analyze multiscale gradients across the urban landscape. We specifically consider implications for racial and ethnic exposure disparities. We find that dense, urban neighborhoods exhibit peaks that vary by pollutant in both location and magnitude, reflecting complex interactions among diverse emission sources and urban microenvironments. Despite high-magnitude hyperlocal peaks, we find that concentration differences between sampling areas (i.e., among distinct neighborhoods and cities) cause greater average concentrations for people of color. These findings demonstrate the need to consider mitigation policies at multiple urban scales to address environmental inequity.

\section{Results and Discussion}

High Variation in Air Pollution Observed from Hyperlocal to Regional Scales. We create empirical maps of four air pollutants in 13 groupings of neighborhoods that constitute urban districts, cities, or towns (total population: 450,000 ), ranging in land use, urbanization, traffic density, demographic composition, and historical housing policy (SI Appendix, Figs. S1, S2, and S12 and Tables S1-S3). We calculate census block scale concentrations (see Materials and Methods) to best approximate conditions at individual residences given the resolution of demographic data (median block population: 36 people). Hereon, we describe outdoor concentration as "exposure" for block residents, terminology consistent with literature linking health effects to census block estimates $(24,44)$; we acknowledge that individual exposure also depends on multiple other factors (e.g., diurnal activity, indoor infiltration and dynamics, and physiology).

Study area maps (Fig. 1) reveal sharp concentration gradients $(\sim 2$ to $5 \times$ ) within groups of contiguous blocks, as well as marked differences in the range of concentrations within different study areas. In discussing spatial variation, we term gradients among neighboring blocks ( $100 \mathrm{~m})$ as "hyperlocal," variation within each study area $(\sim 1 \mathrm{~km})$ as "local," and variation among study areas $(\sim 10 \mathrm{~km})$ as "regional." Among the four pollutants, NO shows the highest-magnitude hyperlocal peaks, with a typical ratio of $10 \times$ between a peak and local median (SI Appendix, Fig. $\mathrm{S} 4$ and Table S5). BC, $\mathrm{NO}_{2}$, and UFP (peak ratios $3.1 \times, 2.7 \times$, and $2.6 \times$, respectively) exhibit shallower hyperlocal gradients and more diffuse peaks. Complex hyperlocal patterns reflect the combined influence of traffic and point source emissions (e.g., local industry and restaurants) and dispersion and reaction dynamics within the built environment (e.g., street canyons, green spaces). Local elevation of NO concentrations is strongly linked to highways and major arterials, while the influence of highways on $\mathrm{BC}, \mathrm{NO}_{2}$, and UFP varies by study area, with roadways showing the smallest influence on UFP (SI Appendix, Table S6-S9). Differences in hyperlocal patterns among pollutants are reflected in the moderate or low correlations of block-level concentrations in some study areas, especially between $\mathrm{BC}$ and UFP (SI Appendix, Fig. S5). These differences demonstrate the importance of measuring multiple pollutants. Furthermore, these patterns likely differ from those of other important pollutants like fine particulate matter $\left(\mathrm{PM}_{2.5}\right)$ and air toxics, both in location and degree of local and regional variation.

Exposure variation reflects where residents live relative to observed local and regional gradients, although residents may also experience peaks in unpopulated areas during daily activities. We find that the weighting of population within more homogeneous residential areas mildly diminishes the importance of local variation, but both local and regional gradients contribute to the broad range of exposures across the study domain. Fig. 2 shows the full distribution of exposure levels within each study area. Comparing median exposures between the most- and least-polluted study areas, concentrations varied by a factor of $4,5,6$, and 28 for $\mathrm{BC}, \mathrm{NO}_{2}, \mathrm{UFP}$, and $\mathrm{NO}$, respectively, while withinneighborhood interdecile ranges showed variation up to a factor of 4 for $\mathrm{BC}, \mathrm{NO}_{2}$, UFP, and a factor of 19 for NO. Generally, neighborhoods with higher $\mathrm{BC}$ and $\mathrm{NO}$ medians also display a wider range of exposures, while $\mathrm{NO}_{2}$ and UFP ranges remain more consistent across neighborhoods. To partition exposure variability into local and regional components, we decomposed the sumof-squared deviation from the mean (SSD) of each resident versus the study area mean and of all study areas versus the grand mean (SI Appendix, Table S12). We find that local gradients contribute the majority of exposure variation for primary pollutants (NO: $52 \%$ and BC: $63 \%$ of SSD) but the minority for $\mathrm{NO}_{2}$ and UFP (37 and $28 \%$, respectively), two pollutants where secondary processes are a key source. A subset of study areas account for a disproportionate share of local variation. For example, the San Francisco (SF) Financial District and East Oakland (24\% of study population) account for roughly $50 \%$ of local exposure variation for $\mathrm{NO}$ and $\mathrm{BC}$ and $40 \%$ for UFP and $\mathrm{NO}_{2}$. These study areas represent denser urban settings with a greater mix of land uses. A similar comparison of purely spatial units (SSD among blocks, SI Appendix, Tables S10 and S11) finds that local variability is much greater for the primary pollutants $\mathrm{NO}$ and $\mathrm{BC}$ (76 and 79\% of the $\mathrm{SSD}$, respectively) and moderately higher for $\mathrm{NO}_{2}$ and $\mathrm{UFP}\left(\mathrm{NO}_{2}\right.$ : $54 \%$ and UFP: $46 \%$ ).

Our observations show a substantially expanded range of exposure-both between and within neighborhoods - than census block-level $\mathrm{NO}_{2}$ predictions from a national-scale predictive 


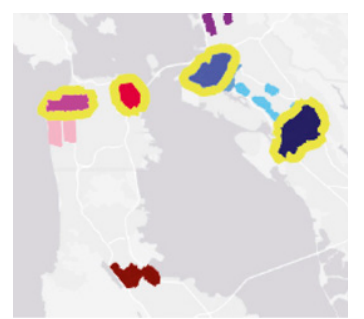

\section{RF \\ District \\ $6.6 \mathrm{~km}^{2}$ pop. 69,000}

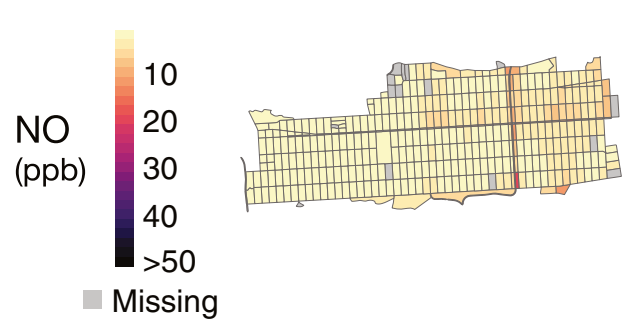

$\begin{array}{ll} & 10 \\ \mathrm{NO}_{2} & 20 \\ (\mathrm{ppb}) & 30 \\ & 40 \\ & >50\end{array}$

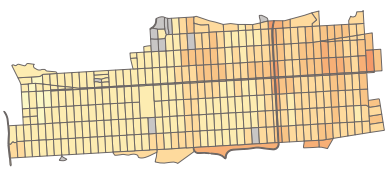

Missing
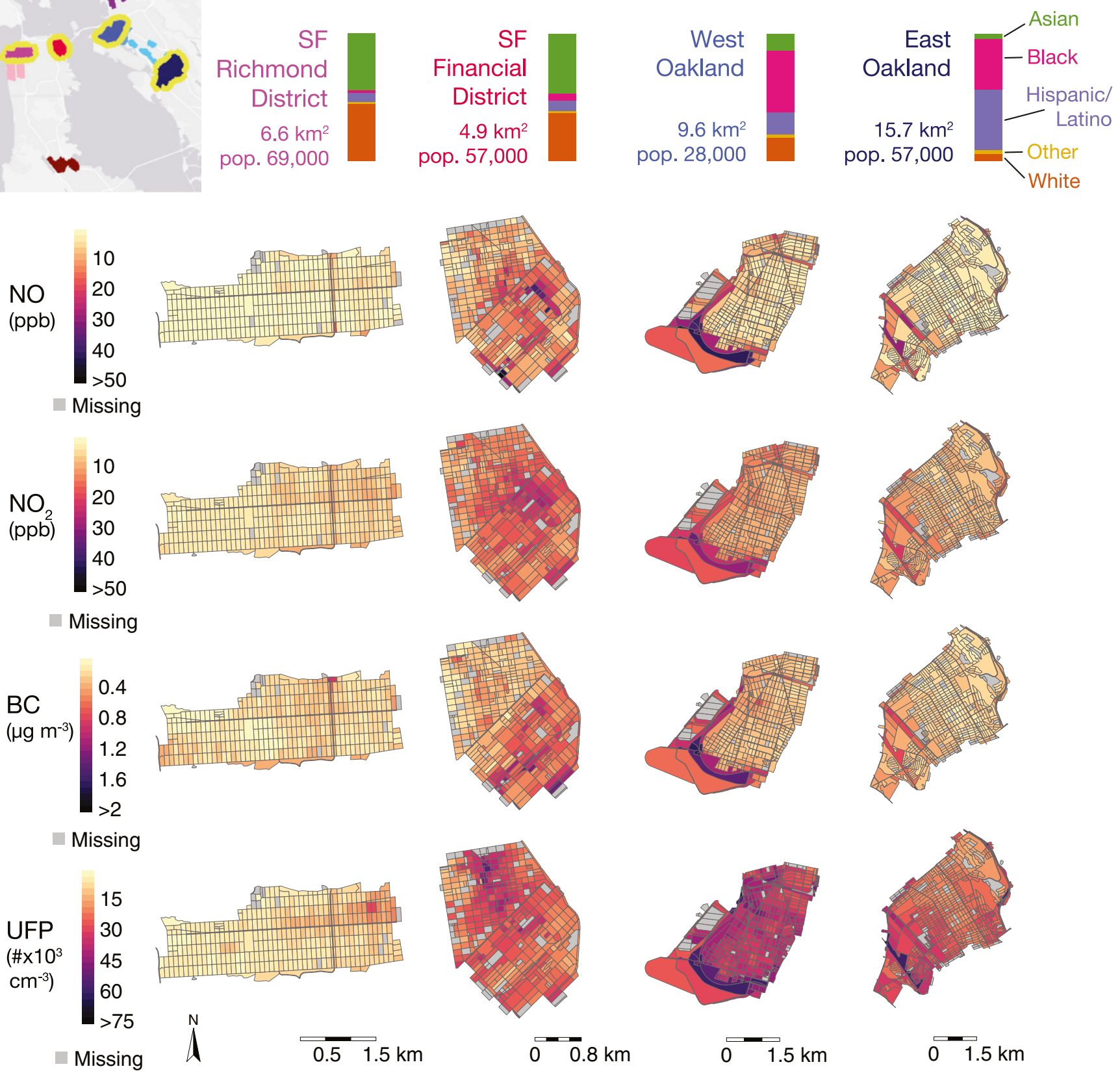

$0 \quad 1.5 \mathrm{~km}$

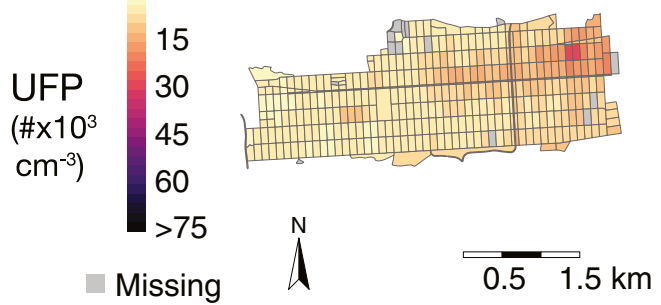

Fig. 1. Daytime median census block pollution levels for four selected study areas, calculated from $\sim 50$ visits to each block. For the bar plots indicating race/ ethnicity, the White, Asian, Black, and Other race groups only include those identifying as non-Hispanic.

model similar to a land use regression (year 2015 integrated empirical-geographic [IEG] model) (17). The national IEG modeled estimates (Fig. 2, gray overlaid plots) show a ratio of 1.6 between highest and lowest neighborhood median exposure compared to 4.6 from mobile monitoring and a total-population interquartile range (IQR) of 2.2 parts per billion (IEG) compared to $6.1 \mathrm{ppb}$ from mobile monitoring data (SI Appendix, Fig. S6). This result suggests that the national IEG model may miss some localized influences and may underestimate total population disparity and, by extension, the potential range of health risks. We find that the IEG model predicts higher median and mean exposure (2.8 ppb [36\%] and $2.5 \mathrm{ppb}$ [30\%] higher, respectively) across the total population, compared to mobile monitoring data. No NO, BC, or UFP data at the same spatial resolution were publicly available at the time of this analysis (45).
Systematically Higher Concentrations for Black and Hispanic/Latino Groups Driven by Regional Variation. A stratification of pollutant exposure by study area and US Census-reported race and ethnicity (Fig. 3) reveals both a wide range in the measures of air pollution within each racial/ethnic group and systematic differences in exposure among groups. Fig. 3 highlights the role of regional demographic patterns in shaping the distribution of exposure across members of each racial/ethnic group. Study areas with a greater share of a given race/ethnicity are represented by larger areas within the shape of the distribution. For example, exposure for the Asian community within our study areas is dominated by neighborhoods in San Francisco (shades of pink), while exposure for Black residents is almost entirely determined by concentrations in Oakland neighborhoods (shades of blue). These aggregate exposure profiles reveal an overall pattern of racial/ethnic disparities: 

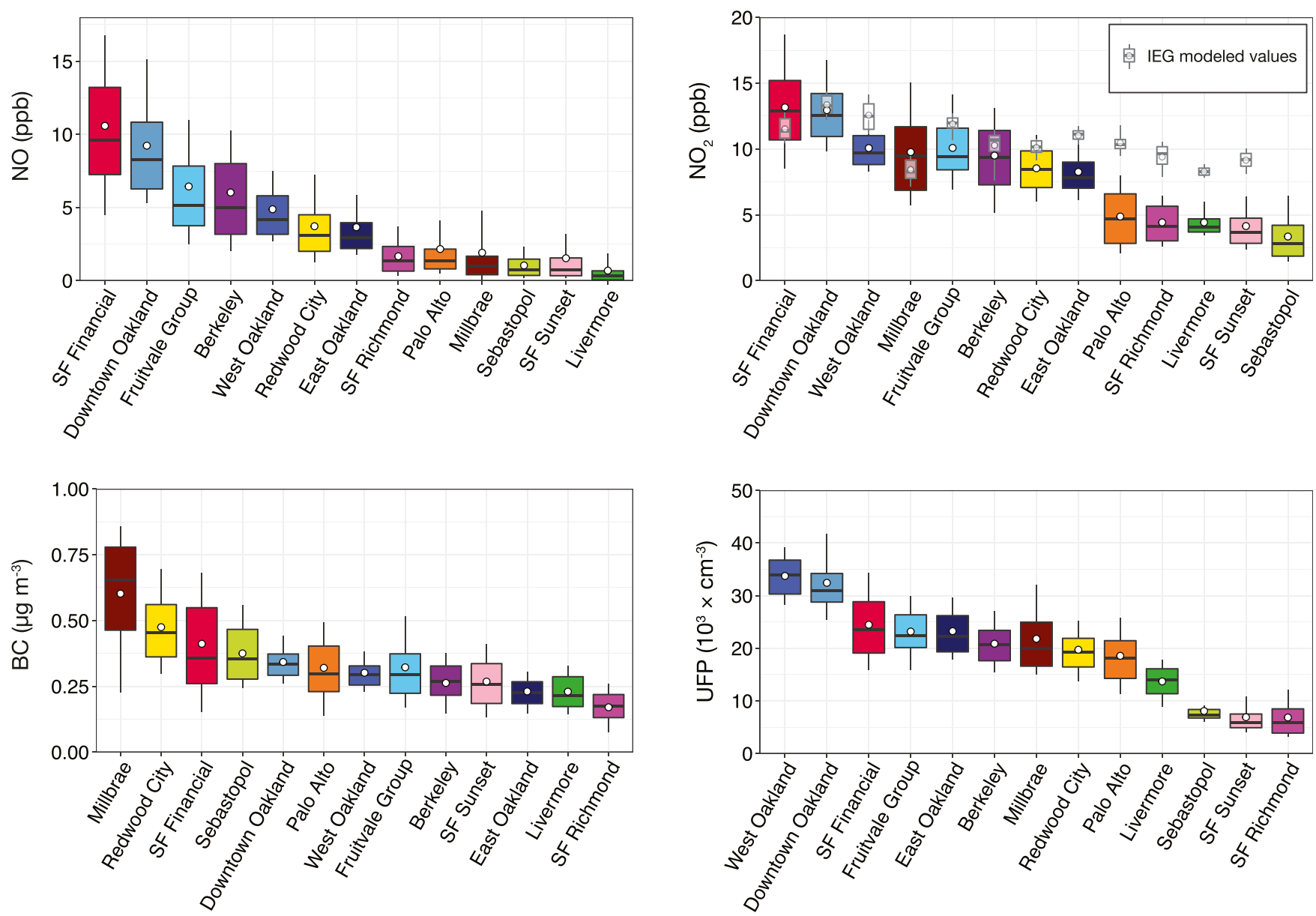

Fig. 2. Exposure distributions by neighborhood, based on daytime, weekday census block concentrations. Areas are shown in order of descending median concentration. Whisker ends represent 10th and 90th percentiles, box boundaries represent upper and lower quartiles, the center bar marks the median, and the circle represents the mean. Gray box plots in the $\mathrm{NO}_{2}$ panel represent modeled exposure estimates from a national-scale IEG regression model (17).

higher concentration ranges in predominantly Black and Hispanic/ Latino neighborhoods result in higher mean exposure for those groups. Notably, many of the neighborhoods with the highest average pollution exposures in our measurement dataset were subjected to overtly racially discriminatory housing policies (i.e., redlining) during the twentieth century (SI Appendix, Fig. S12).

At the local level, there is wide overlap among the exposure distributions of each racial/ethnic group (SI Appendix, Fig. S7). While study areas do show weak patterns of local disparitycensus blocks with majority White residents showed modestly lower concentrations than blocks with majority Black and Hispanic/Latino residents within the same study area-there are not universal trends. In a few instances, a specific community shows exceptionally high exposure, as exemplified by the Asian populations of Downtown Oakland (all pollutants) and the SF Financial District (UFP). Both districts include densely populated corridors occupied by people of Asian descent.

In contrast, we find substantial disparity arising from regional pollution gradients combined with the high intermunicipal racial segregation in the Bay Area (46). On average, the White population is exposed to lower $\mathrm{NO}, \mathrm{NO}_{2}$, and UFP than other groups, with a median exposure 16 to $27 \%$ below the total-population median, while medians for the Black and Hispanic/Latino populations are higher by 8 to $30 \%$ depending on pollutant (Fig. $4 A$ and SI Appendix, Table S13). The spatial detail provided by our method reveals nuance in disparity patterns beyond differences in medians. Fig. $4 B$ illustrates the weighting of each racial/ethnic group within total-population exposure deciles. Overall, the White population is strongly overrepresented in the lowest deciles of the concentration distributions. The Asian population is overrepresented at the extremes, with the high end driven by the previously mentioned communities in Downtown Oakland and the SF Financial District and the low end driven by the Richmond and Sunset Districts. The Black and Hispanic/Latino populations are strongly underrepresented at the low end and concentrated toward the higher deciles, giving rise to higher average exposures for those groups. Apart from distinctly higher ranges of $\mathrm{NO}_{2}$ and UFP exposure among Black and Hispanic/Latino populations, the range of exposures within racial/ethnic groups tends to be large compared with the range among groups. This finding holds especially for the Asian population (Fig. $4 A$ ), which is bimodally distributed between some of the cleanest (coastal) and most polluted (downtown) areas.

Within our study domain, the national IEG model also predicts lowest mean exposure for the White population and highest for the Black population, with moderately higher exposure for the Hispanic/Latino population (SI Appendix, Fig. S5). However, because of the narrower band of exposure estimates, IEGmodeled disparity differs from observations. The IEG model predicts a similar magnitude of exposure disparity between the population median and medians for those of Black and Hispanic/ Latino descent, but a smaller difference for the White population, and does not show a disproportionate share of people of Asian descent in the highest-exposure categories. Thus, modeled 

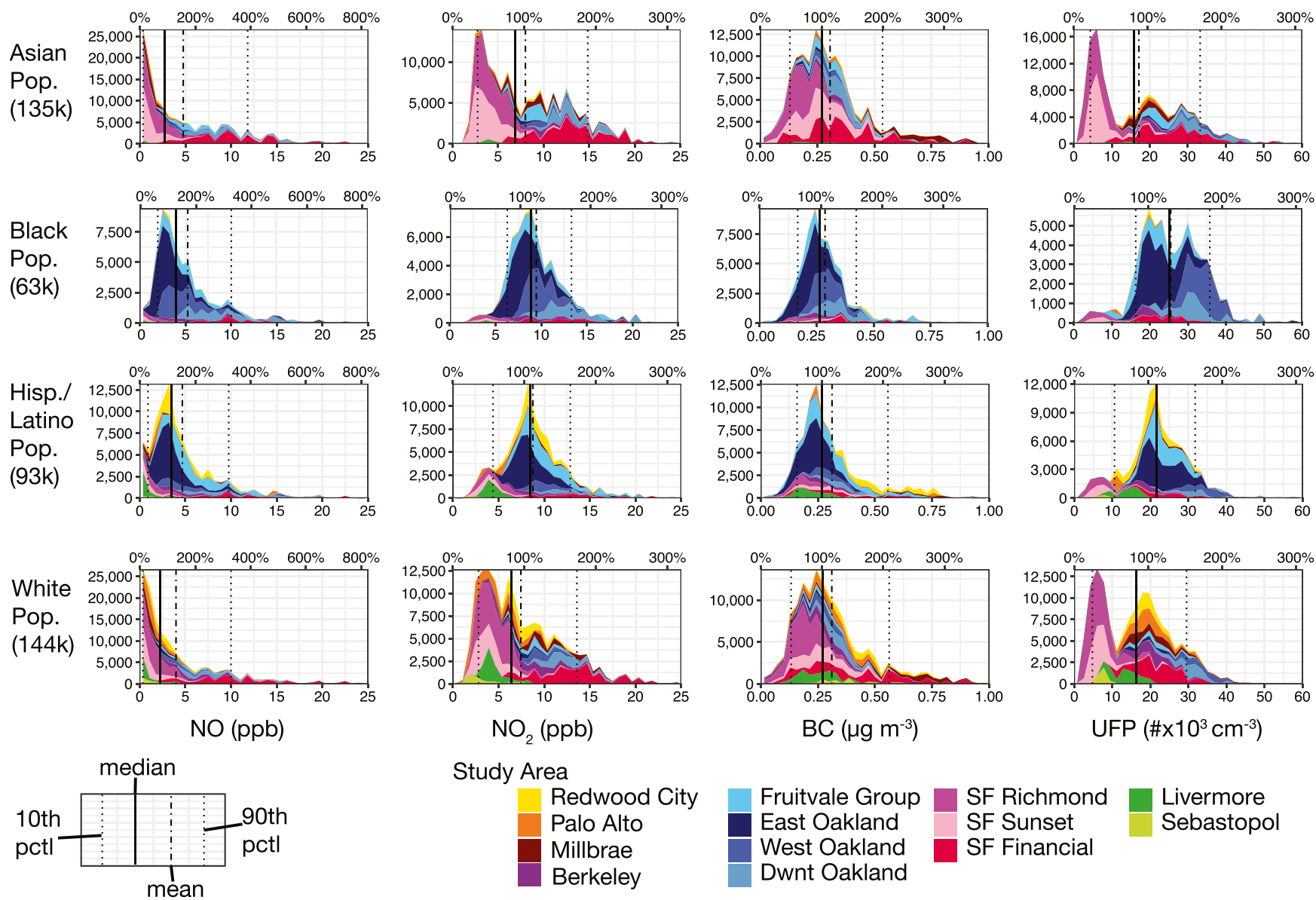
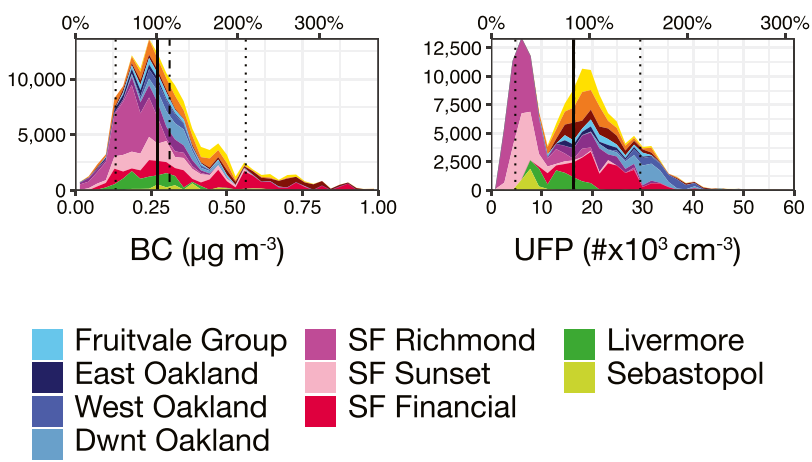

Livermore

Sebastopol
SF Sunset SF Financial
Palo Alto

Millbrae

Berkeley

Fig. 3. Distribution of potential exposure stratified by race/ethnicity (not shown is the "Other" racial category, population: 10,000). The White, Asian, Black, and Other race groups only include those identifying as non-Hispanic. Height on the $y$-axis indicates the population at a given concentration level summed over all study areas. Because of significant differences in the racial and ethnic composition of each neighborhood, concentration distributions in some study areas contribute much more to specific race/ethnicities (e.g., East Oakland for the Hispanic/Latino population, West Oakland for the Black population). Vertical lines show the indicated statistics (mean, median, 10th, and 90th percentiles) for each race/ethnicity. Corresponding relative disparity values are provided in SI Appendix, Table S13.

disparity may miss an important dimension of racial/ethnic exposure disparity.

Our conclusions come with several caveats. First, mobile monitoring was conducted during daytime, weekday hours. Concentrations and spatial gradients may differ during nighttime and weekend hours due to changes in emission patterns and atmospheric dynamics $(28,33)$. Second, we assess here the implications of temporally averaged patterns, which are associated with chronic health effects. However, concentrations and spatial patterns show strong diurnal and seasonal variation that may have acute effects. While we believe that we sampled representative conditions (see Materials and Methods), temporal pollution dynamics could add another dimension to exposure disparity patterns. For this reason, we recommend that in-depth neighborhood monitoring include continuous fixed-site measurements in addition to mobile measurements (28). Third, we found that our conclusions are likely sensitive to the choice of neighborhoods, so we cannot assert that the disparity patterns we found are fully representative of conditions throughout the Bay Area (i.e., in unsampled neighborhoods). Finally, disparities in personal exposure to pollutants of outdoor origin depend not only on fine-scale patterns of outdoor air pollution-as measured here-but also personal mobility and the penetration of pollutants into the indoor environment. Future developments in measurement and modeling could usefully refine understanding of these dimensions of disparity. However, this study affirms that assessments of racial/ethnic air pollution disparities in the United States should include a broad range of representative neighborhoods that capture the varied experiences of those within each racial/ethnic group, considering the context of historic and present day municipal-level segregation.

Implications for Future Exposure Assessments and Environmental Justice Research. The problem of disparate exposures to environmental pollution has become the focus of renewed policy interest in the United States. Full-coverage mobile monitoring of air pollution can support policy advances in several ways. Highintensity mobile monitoring can reveal sources of localized hotspots (e.g., warehouses, metals processing, or restaurant clusters) that may be the subject of community concern but are not always detected by conventional monitoring (47). More broadly, the range and spatial variability of exposures revealed by mobile monitoring-not generally provided by conventional modeling and regulatory measurement networks-support the development of community-focused plans to improve air quality.

Our findings highlight the importance of identifying both highly localized patterns and intraurban disparity for addressing environmental injustice. We performed a set of stylized sensitivity analyses, described in SI Appendix, to characterize the degree to 

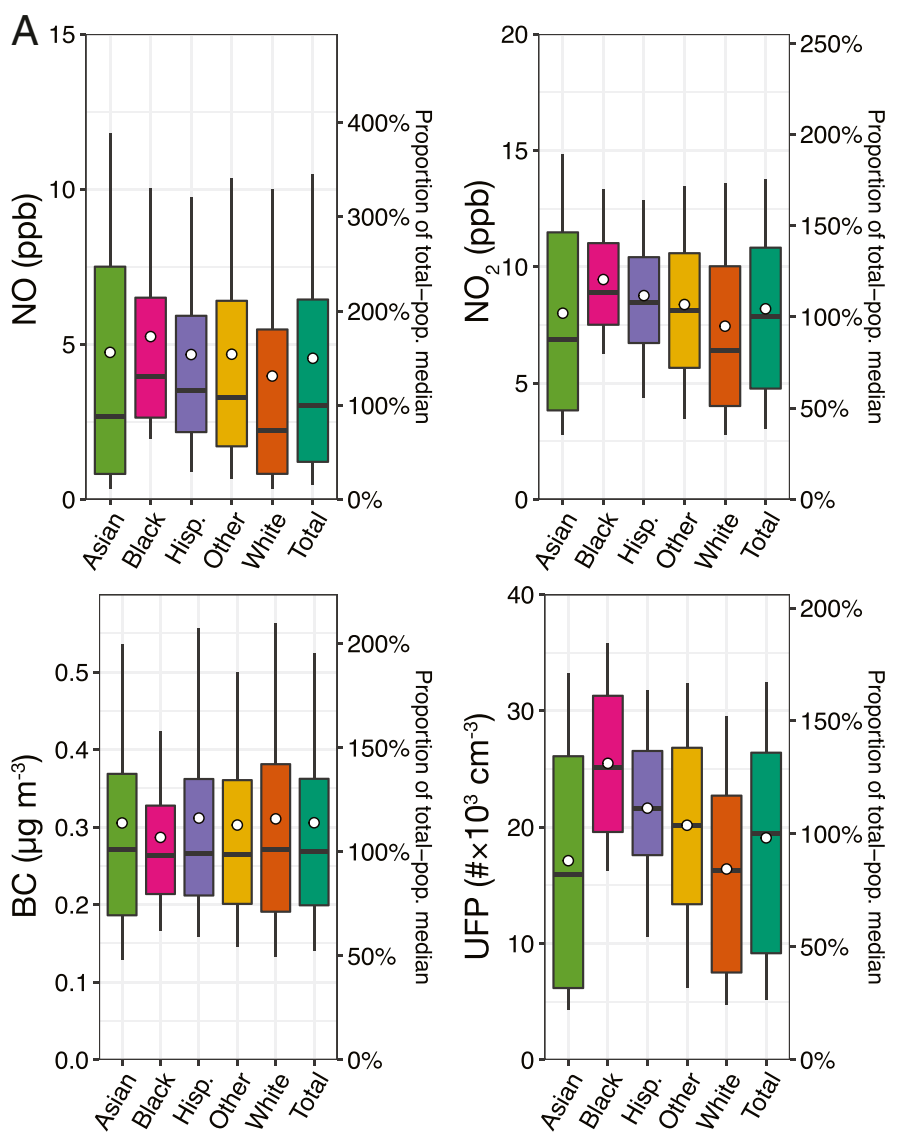
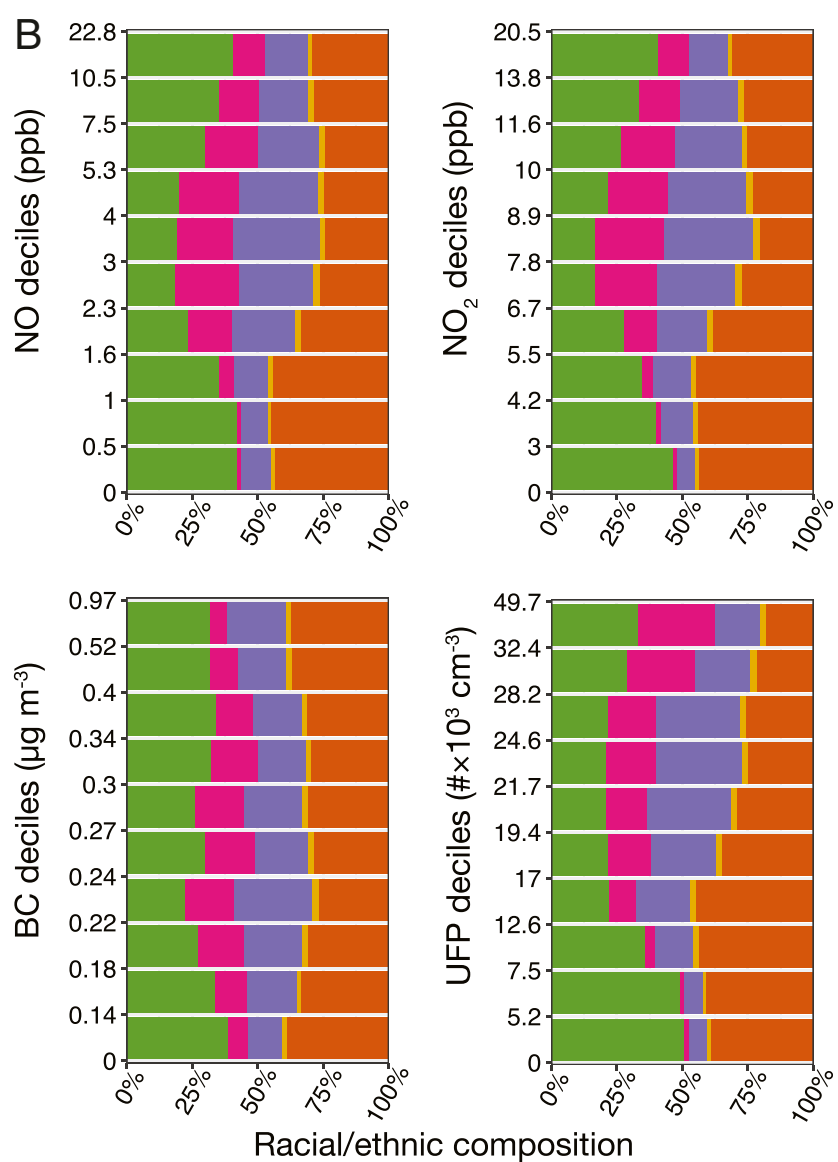

Fig. 4. Variation in total exposure distributions by race/ethnicity group. The distributions shown in the box-and-whisker plots $(A)$ include the median (central bar), mean (white circle), upper and lower quartiles (box boundaries), and upper and lower 90th and 10th percentiles (whiskers). The division of the totalpopulation exposure distribution into concentration deciles $(B)$ shows the division of the population within the decile by race/ethnicity, with decile boundary concentrations indicated on the $y$-axis and the racial/ethnic color key provided by the box-and-whisker plots. The White, Asian, Black, and Other race groups only include those identifying as non-Hispanic.

which hyperlocal, local, and regional air pollution control interventions might help reduce racial/ethnic exposure disparities within the San Francisco Bay Area. Consistent with our findings that between-neighborhood patterns are the principal driver of racial/ethnic disparities, interventions that only eliminate withinneighborhood hotspots may not substantially reduce systematic disparity, although such interventions address the concentration extremes often experienced disproportionately by people of color. In contrast, interventions that reduce the differences in background concentrations among neighborhoods have higher potential to address population-wide racial/ethnic exposure disparities (SI Appendix). Higher-pollution areas often contain a variety of features that individually cause hyperlocal peaks and as a group contribute to elevated local background concentrations. Some examples of these features include greater heavy-duty vehicle traffic, adjacent high-emissions areas like ports and railyards, a high density of industrial operations, and a lack of green space. A community-centered approach to policymaking can account for issues at both hyperlocal and local scales by first identifying a suite of neighborhood features that contribute to higher pollution and then seeking local, regional, or state support for targeted interventions. Such interventions may include incentives and infrastructure to support zero-emission vehicles, accelerated emission control retrofits for point sources, and city planning to restrict new industrial facilities in disproportionately burdened communities. The differences in disparity patterns among pollutants further underscores the importance of multisource control policies. This community-centered approach is exemplified by the West Oakland "Owning Our Air" plan coauthored by the Bay Area Air Quality Management District, the West Oakland Environmental Indicators project, and a community-based steering committee and the broader Community Air Protection Program (AB617) in California, which includes West Oakland among 15 areas chosen for targeted interventions $(47,48)$.

Continued monitoring is necessary for evaluating policy effectiveness. Regional monitoring strategies may benefit from the multiscale approach of this study, as we identified some areas for which a single "background" monitor would represent neighborhood exposure within reasonable bounds, especially for secondary pollutants. Further investigation of the association of peaks and regional variation with specific emission sources and land uses is a useful direction for future research. We hypothesize that expanding this approach to other urban or rural settings would likely reveal different but similarly complex patterns and advance our understanding of the interaction between demographic patterns, pollution sources, and the built environment.

Environmental policy efforts in the coming years will increase the emphasis on addressing systemic racial/ethnic disparities in air pollution exposure. We illustrate here how environmental justice concerns arise from the confluence of multiscale patterns of emissions, concentrations, and populations within urban landscapes. Future efforts to address disparities will benefit from recent advances in both extensive fine-scale measurement-as we have demonstrated here-as well as source-oriented models 
that identify the specific causes and drivers of exposure disparities. Broader solutions to environmental injustice will also involve greater partnership between scientists and community organizations, new approaches to synthesizing and communicating highresolution data, and innovations in inclusive policy making.

\section{Materials and Methods}

Measurement Methods. Mobile monitoring was conducted with two Google Street View vehicles equipped with the Aclima mobile platform, which used research-grade instrumentation to provide $1-\mathrm{Hz}$ measurements of $\mathrm{BC}, \mathrm{NO}$, $\mathrm{NO}_{2}$, and UFP (SI Appendix, Table S14), and Global Positioning System coordinates with a nominal precision of $\sim 1 \mathrm{~m}$. Detailed description of measurement and calibration protocol is contained in Apte et al. (21). The inlet and sampling manifold, mounted on the roof of each mobile laboratory, was designed to minimize self-sampling (measurements of the vehicle's own exhaust) as well as particle- and gas-phase sampling losses, with an estimated particle sampling loss of $<5 \%$. Raw measurement data were reviewed and processed to exclude periods of poor instrument performance (49). Data collection occurred over 32 mo from May 2015 through December 2017. The average nonhighway driving speed of $9 \mathrm{~m} \cdot \mathrm{s}^{-1}$ provides a nominal spatial resolution of $\sim 10 \mathrm{~m}$

Study areas were repeatedly visited on a rotating schedule designed to assess long-term average concentrations indicative of typical weekday, daytime conditions. During a visit, the driver would follow a Google Street View-based driving protocol to visit every road segment within the neighborhood at least once, driving with the normal flow of traffic. For large study areas, a subunit would be assigned for full coverage in a single day's driving, with full sampling occurring over multiple days. Visits to each area were distributed over different times of day and different seasons. Incidental temporal sampling bias was assessed using fixed-site regulatory monitors located in five of the sampling areas (SI Appendix)

Study Areas and Demographic Data. In total, 13 urban districts, cities, and towns ranging in size from 2.4 to $15.7 \mathrm{~km}^{2}$ (total: $93 \mathrm{~km}^{2}$ ) were selected as study areas to provide a range of land uses (e.g., industrial, commercial, dense residential, and light residential), share of open or green space, traffic density, demographic composition, and historical housing policy (SI Appendix, Figs. S1, S2, S12, and S13 and Tables S1-S4). Study areas were distributed within the counties of San Francisco, Alameda, San Mateo, and Santa Clara in the San Francisco Bay Area, with one background location in Sonoma County (SI Appendix, Fig. S5 and Table S6). Sampling areas include between 95 and 930 census blocks (median: 447), depending on population density and spatial extent (SI Appendix, Table S4).

Population data from the US Census Bureau were retrieved from the IPUMS National Historical Geographic Information System for the year 2010, the most recent year for which block-level data are available (50). Using the racial/ethnic designations provided by the US Census, we categorize all individuals identifying as Latino and/or Hispanic in one group, and then categorize non-Hispanic individuals by race: Asian, Black, White, and "Other," including those of Native American, Pacific Islander, multiracial, or other racial identity. The racial composition of our study population is broadly representative of the Bay Area as a whole, although it includes more neighborhoods with a high proportion of Black residents (SI Appendix, Fig. S2).

Data Processing. Concentrations for each block are calculated as the median of surrounding roads, typically located within 50 to $100 \mathrm{~m}$ from the block center point (SI Appendix, Fig. S3). The geographic assignment of on-road measurements to census blocks involved a two-step process. First, concentrations were calculated for road segments: the raw longitude and latitude values associated with each $1-\mathrm{Hz}$ measurement were "snapped" to $30 \mathrm{~m}$ road segment points, as described by Apte et al. (21). To give equal weight to each pass of a road segment and minimize the influence of transient extreme events (e.g., vehicle plumes), measurements at each road segment were averaged per drive pass: all consecutive measurements made at a single road segment were summarized as a single mean value, following methods described in Messier et al. (43). Long-term concentrations at each road segment were calculated as the median of those drive pass means, with the median statistic chosen to further reduce the influence of outlier events.

Second, census block concentrations were calculated as the median of concentrations at every adjacent or intersecting $30-\mathrm{m}$ road segment, using 10-m buffer to capture road segments a small distance from the census block edge. This dataset of census block concentrations and demographic data has been posted to an online repository (51). Blocks vary in size and shape but typically cover an area of $\sim 0.01 \mathrm{~km}^{2}$ and are surrounded by roads, with a median perimeter of $447 \mathrm{~m}$. Accordingly, census block estimates integrated measurements from 15 to 20 road segments (SI Appendix, Fig. S3). In some cases-near highways and strong point sources-pollution gradients may vary over finer spatial scales than those captured by census block spatial units $(\sim 110 \mathrm{~m})$. However, the integration of multiple road segments provides an increase in total number of visits and total sampling time per spatial unit, which reduces sampling error and measurement uncertainty (52). While on-road measurements are not a perfect approximation of concentrations throughout a census block, a previous comparison of onand near-road measurements in West Oakland showed no evidence of bias in on-road concentrations due to increased proximity to on-road emissions (52). The general spatial representativeness of on-road measurements holds especially in low-traffic residential neighborhoods with mixed wind conditions (34).

Uncertainty and Sensitivity Analyses. Because of the temporal sparsity of mobile measurements relative to fixed-site monitoring, it is critical to consider both the uncertainty inherent in a small sample size and the potential for temporal bias based on the days and times of day sampled. To mitigate temporal bias from lower sampling rates, we imposed the following standards for all data used in this analysis: Road segments were included if valid measurements were made on at least $3 \mathrm{~d}$, and census blocks were only included if they integrated measurements from three or more road segments and included a total of $100 \mathrm{~s}$ or more of valid sampling data. The median cumulative sampling time of each block was $19 \mathrm{~min}$, collected during a median of 47 unique visits over $20 \mathrm{~d}$.

A bootstrap resampling technique, described in SI Appendix, was used to estimate uncertainty at different sampling intensities. The characteristic sampling intensity for each study area is provided in SI Appendix, Table S4. A Monte Carlo subsampling analysis of an exceptionally highly sampled area (West Oakland) shows that with moderate sampling intensity (each block visited 10 to $20 \mathrm{~d}$ ) provides a $90 \% \mathrm{Cl}$ around the population-weighted median concentration of approximately $\pm 20 \%$ for $\mathrm{NO}_{2}$ and UFP, $\pm 30 \%$ for $\mathrm{BC}$, and $\pm 40 \%$ for NO, with Cls that are positively skewed (SI Appendix, Table S15). At higher sampling intensity ( 20 to $40 \mathrm{~d}$ ), the $90 \% \mathrm{Cl}$ is $\pm 20 \%$ for the medians of all pollutants. Considering the influence of sampling intensity on estimates of within-neighborhood variability, we find that even at high sampling intensity the interdecile range is overestimated in $95 \%$ of simulations, affecting both estimates of local variance and the total-population variance. Despite this effect, we find that study areas that accounted for large portions of SSD for $\mathrm{NO}, \mathrm{NO}_{2}$, and UFP included some of the mostsampled (Downtown Oakland and Berkeley) as well as moderately sampled areas (SF Financial and Fruitvale Group; SI Appendix, Table S4). Nevertheless, this finding serves as a caution against overinterpreting variation in less-sampled areas.

Diurnal sampling bias metrics show that sampling of $\mathrm{NO}_{2}, \mathrm{BC}$, and UFP was broadly representative $( \pm 15 \%)$ of daytime conditions, while the distribution of hourly measurements resulted in a systematic low bias in NO concentrations (SI Appendix, Table S16). Error tended to be in the same direction across all study areas (e.g., 12 out of 13 are biased $\sim 10 \%$ lower for BC) and thus had a small effect on the relative rankings of concentrations among study areas (SI Appendix, Fig. S8). Annual representativeness ratios show less consistent patterns across study areas, and the application annual adjustment factor results in minor changes in the relative rankings of $\mathrm{NO}_{2}$ and $\mathrm{BC}$ among study areas and increases the ranges of NO and UFP exposure distributions among high concentration study areas. Overall, these findings are robust against the effects of temporal bias.

Data Availability. A dataset including concentrations and demographics by census block has been deposited in figshare $(10.6084 / \mathrm{m} 9$.figshare.15070314) (51). All other study data are included in the article and/or supporting information.

ACKNOWLEDGMENTS. We gratefully acknowledge the contributions of S. Gani, R. Alvarez, M. Harris, M. Gordon, B. Beveridge, D. Holstius, P. Martien, K. Tuxen-Bettman, and the Google Street View and Aclima mobile platform team. Funding was provided by the Environmental Defense Fund and the Health Effects Institute, an organization jointly funded by the US Environmental Protection Agency (EPA) (Assistance Award No. R-82811201) and certain motor vehicle and engine manufacturers. This publication was developed as part of the Center for Air, Climate and Energy Solutions, which was supported under Assistance Agreement No. R835873 awarded by the US EPA. It has not been formally reviewed by the EPA. The views expressed in this document are solely those of authors and do not necessarily reflect those of the Agency. The EPA does not endorse any products or commercial services mentioned in this publication. 
1. A. A. Karner, D. S. Eisinger, D. A. Niemeier, Near-roadway air quality: Synthesizing the findings from real-world data. Environ. Sci. Technol. 44, 5334-5344 (2010).

2. P. Gu et al., Intracity variability of particulate matter exposure is driven by carbonaceous sources and correlated with land-use variables. Environ. Sci. Technol. 52, 11545-11554 (2018).

3. Y. Wang et al., Spatial decomposition analysis of NO2 and PM2.5 air pollution in the United States. Atmos. Environ. 241, 117470 (2020)

4. J. G. Su, M. Jerrett, R. Morello-Frosch, B. M. Jesdale, A. D. Kyle, Inequalities in cumulative environmental burdens among three urbanized counties in California. En viron. Int. 40, 79-87 (2012)

5. D. Houston, W. Li, J. Wu, Disparities in exposure to automobile and truck traffic and vehicle emissions near the Los Angeles-Long Beach port complex. Am. J. Public Health 104, 156-164 (2014)

6. M. R. Jones et al., Race/ethnicity, residential segregation, and exposure to ambient air pollution: The Multi-Ethnic Study of Atherosclerosis (MESA). Am. J. Public Health 104 2130-2137 (2014)

7. R. Morello-Frosch, B. M. Jesdale, Separate and unequal: Residential segregation and estimated cancer risks associated with ambient air toxics in U.S. metropolitan areas. Environ. Health Perspect. 114, 386-393 (2006).

8. N. P. Nguyen, J. D. Marshall, Impact, efficiency, inequality, and injustice of urban air pollution: Variability by emission location. Environ. Res. Lett. 13, 024002 (2018).

9. C. W. Tessum et al., Inequity in consumption of goods and services adds to racialethnic disparities in air pollution exposure. Proc. Natl. Acad. Sci. U.S.A. 116, 6001-6006 (2019).

10. C. W. Tessum et al., $\mathrm{PM}_{2.5}$ polluters disproportionately and systemically affect people of color in the United States. Sci. Adv. 7, eabf4491 (2021).

11. A. J. Cohen et al., Estimates and 25-year trends of the global burden of disease attributable to ambient air pollution: An analysis of data from the Global Burden of Diseases Study 2015. Lancet 389, 1907-1918 (2017).

12. D. E. Schraufnagel et al., Air pollution and noncommunicable diseases: A review by the forum of international respiratory societies' environmental committee, part 2: Air pollution and organ systems. Chest 155, 417-426 (2019).

13. X. Wu, R. C. Nethery, M. B. Sabath, D. Braun, F. Dominici, Air pollution and COVID-19 mortality in the United States: Strengths and limitations of an ecological regression analysis. Sci. Adv. 6, eabd4049 (2020)

14. A. van Donkelaar, R. V. Martin, C. Li, R. T. Burnett, Regional estimates of chemical composition of fine particulate matter using a combined geoscience-statistical method with information from satellites, models, and monitors. Environ. Sci. Technol. 53, 2595-2611 (2019).

15. M. S. Hammer et al., Global estimates and long-term trends of fine particulate matter concentrations (1998-2018). Environ. Sci. Technol. 54, 7879-7890 (2020).

16. G. Hoek, Methods for assessing long-term exposures to outdoor air pollutants. Curr. Environ. Health Rep. 4, 450-462 (2017).

17. S.-Y. Kim et al., Concentrations of criteria pollutants in the contiguous U.S., 1979 2015: Role of prediction model parsimony in integrated empirical geographic regression. PLoS One 15, e0228535 (2020).

18. J. Peters, J. Theunis, M. V. Poppel, P. Berghmans, Monitoring $\mathrm{PM}_{10}$ and ultrafine particles in urban environments using mobile measurements. Aerosol Air Qual. Res. 13, 509-522 (2013).

19. A. P. Patton et al., Spatial and temporal differences in traffic-related air pollution in three urban neighborhoods near an interstate highway. Atmos. Environ. 99, 309-321 (2014).

20. N. Baldwin et al., Factors affecting pollutant concentrations in the near-road environment. Atmos. Environ. 115, 223-235 (2015).

21. J. S. Apte et al., High-resolution air pollution mapping with Google Street View cars: Exploiting big data. Environ. Sci. Technol. 51, 6999-7008 (2017).

22. P. Deshmukh et al., Identifying air pollution source impacts in urban communities using mobile monitoring. Sci. Total Environ. 715, 136979 (2020).

23. G. Oberdörster, A. Elder, A. Rinderknecht, Nanoparticles and the brain: Cause for concern? J. Nanosci. Nanotechnol. 9, 4996-5007 (2009).

24. S. E. Alexeeff et al., High-resolution mapping of traffic related air pollution with Google street view cars and incidence of cardiovascular events within neighborhoods in Oakland, CA. Environ. Health 17, 38 (2018)

25. E. F. Kirrane et al., A systematic review of cardiovascular responses associated with ambient black carbon and fine particulate matter. Environ. Int. 127, 305-316 (2019).

26. HEI Review Panel on Ultrafine Particles, "Understanding the health effects of ultrafine particles" (2013) (4 October 2020).
27. R. U. Shah et al., Socio-economic disparities in exposure to urban restaurant emissions are larger than for traffic. Environ. Res. Lett. 15, 114039 (2020)

28. J. J. Caubel, T. E. Cados, C. V. Preble, T. W. Kirchstetter, A distributed network of 100 black carbon sensors for 100 days of air quality monitoring in West Oakland, California. Environ. Sci. Technol. 53, 7564-7573 (2019).

29. R. M. Brice, J. F. Roesler, The exposure to carbon monoxide of occupants of vehicle moving in heavy traffic. J. Air Pollut. Control Assoc. 16, 597-600 (1966).

30. G. A. Petersen, R. H. Sabersky, Measurements of pollutants inside an automobile. J. Air Pollut. Control Assoc. 25, 1028-1032 (1975).

31. D. Westerdahl, S. Fruin, T. Sax, P. M. Fine, C. Sioutas, Mobile platform measurements of ultrafine particles and associated pollutant concentrations on freeways and residential streets in Los Angeles. Atmos. Environ. 39, 3597-3610 (2005).

32. K. H. Kozawa, S. A. Fruin, A. M. Winer, Near-road air pollution impacts of goods movement in communities adjacent to the Ports of Los Angeles and Long Beach. Atmos. Environ. 43, 2960-2970 (2009).

33. S. Hu et al., Observation of elevated air pollutant concentrations in a residential neighborhood of Los Angeles California using a mobile platform. Atmos Environ (1994) 51, 311-319 (2012)

34. I. Levy et al., Elucidating multipollutant exposure across a complex metropolitan area by systematic deployment of a mobile laboratory. Atmos. Chem. Phys. 14, 7173-7193 (2014).

35. S. Hankey, J. D. Marshall, On-bicycle exposure to particulate air pollution: Particle number, black carbon, PM2.5, and particle size. Atmos. Environ. 122, 65-73 (2015).

36. L. M. Zwack, C. J. Paciorek, J. D. Spengler, J. I. Levy, Characterizing local traffic contributions to particulate air pollution in street canyons using mobile monitoring techniques. Atmos. Environ. 45, 2507-2514 (2011).

37. G. S. W. Hagler et al., Field investigation of roadside vegetative and structural barrie impact on near-road ultrafine particle concentrations under a variety of wind conditions. Sci. Total Environ. 419, 7-15 (2012).

38. R. W. Baldauf et al., Air quality variability near a highway in a complex urban environment. Atmos. Environ. 64, 169-178 (2013).

39. J. Steffens et al., Near-port air quality assessment utilizing a mobile measurement approach. Atmos. Pollut. Res. 8, 1023-1030 (2017)

40. H. L. Brantley et al., Characterization of spatial air pollution patterns near a large railyard area in Atlanta, Georgia. Int. J. Environ. Res. Public Health 16, 535 (2019).

41. R. U. Shah et al., High spatial resolution mapping of aerosol composition and sources in Oakland, California using mobile aerosol mass spectrometry. Atmos. Chem. Phys. 10.5194/acp-2018-703 (2018)

42. Z. Li, J. C. H. Fung, A. K. H. Lau, High spatiotemporal characterization of on-road PM $\mathrm{PM}_{2.5}$ concentrations in high-density urban areas using mobile monitoring. Build. Environ. 143, 196-205 (2018)

43. K. P. Messier et al., Mapping air pollution with Google Street View cars: Efficient approaches with mobile monitoring and land use regression. Environ. Sci. Technol. 52 12563-12572 (2018)

44. S. Weichenthal et al., Within-city spatial variations in ambient ultrafine particle concentrations and incident brain tumors in adults. Epidemiology 31, 177-183 (2020).

45. V. A. Southerland et al., Assessing the distribution of air pollution health risks within cities: A neighborhood-scale analysis leveraging high-resolution data sets in the Bay Area, California. Environ. Health Perspect. 129, 37006 (2021).

46. Othering \& Belonging Institute, Racial Segregation in the San Francisco Bay Area (2018). https://belonging.berkeley.edu/segregationinthebay. Accessed 20 August 2020.

47. Bay Area Air Quality Management District, West Oakland Environmental Indicator Project, Owning Our Air: The West Oakland Community Action Plan (2019). https:// www.baaqmd.gov/ /media/files/ab617-community-health/west-oakland/100219-files/ owning-our-air-plan-summary-pdf.pdf. Accessed 22 August 2021.

48. CARB, California Air Resources Board (Community Air Protection Blueprint, 2018).

49. M. Lunden, B. LaFranchi, Metadata for Aclima-Google California AQ mapping dataset" (2017). https://docs.google.com/document/d/1qOR8j4ORSV9VhaAUVuLDnf9nfLh RGbd7rCycXh3U3s. Accessed 9 November 2020.

50. S. Manson, J. Schroeder, D. Van Riper, S. Ruggles, IPUMS National Historical Geographic Information System: Version 14.0 [Database] (IPUMS, 2019).

51. S. E. Chambliss et al., Bay Area mobile monitoring multi-pollutant block Medians. Figshare. https://figshare.com/articles/dataset/Bay_Area_Mobile_Monitoring_Multipollutant_Block_Medians/15070314. Deposited 30 July 2021

52. S. E. Chambliss et al. Comparison of mobile and fixed-site black carbon measurements for high-resolution urban pollution mapping. Environ. Sci. Technol. 54, 7848-7857 (2020). 